\title{
Evaluation of efficacy between Amlexanox 5\% oral paste \& Triamcinolone Acetonide Oral Paste in the Treatment of Recurrent Aphthous Ulcers: A Comparative Study
}

\author{
Molla $\mathrm{NU}^{1}$, Bhuian $\mathrm{I}^{2}$, Hossain $\mathrm{S}^{3}$, Wahab $\mathrm{A}^{4}$, Nayeem $\mathrm{A}^{5}$
}

\begin{abstract}
Background: Recurrent aphthous ulcers typically are multiple round or ovoid ulcers with a circumscribed margin, erythematous halo and a yellow or gray floor. Objective: The purpose of the study to compare the efficacy between Amlexanox 5\% oral paste \& Triamcinolone Acetonide oral paste in the treatment of recurrent aphthous ulcers. Methodology: This was a prospective, open randomized clinical trial of forty patients with recurrent aphthous ulcer. Total 40 cases having recurrent aphthous ulcers were selected for the study during the period of September 2008 to March 2009 in the Department of Dermatology \& Venereology \& Department of Dentistry of Bangabandhu Sheikh Mujib Medical University(BSMMU) \&Shaheed Suhrawardy Medical College \& Hospital(ShSMCH) in Dhaka city . All the cases were diagnosed clinically on the basis by assessing morphology, site \& characters of the lesions. The patients were randomly divided into two equal groups Group-A \& Group- B. Group-A ( $\mathrm{n}=20$ ) was given Amlexanox $5 \%$ oralpaste four times daily for 10 days, and a followup were conducted after 10 days, 4 th week, 8 th week and 12 th week. On the other hand Group- $\mathrm{B}(\mathrm{n}=20)$ was given triamcinolone acetonide oral ointment ( Kenalog in orabase) for three times daily for 10 days. Results: Tingling was a complain of $70 \%$ of Triamcinolone group and $80 \%$ of Amlexanox group. There was no significance difference between groups according to these clinical features. At 10 th day follow up Amlexanox group showed better improvement of pain, although there was no significant difference between two groups at 4th week, 8th week and 12th week follow-up. Marked decreased of mean pain score was observed in the patients of both types of treatment. Mean pain score decreased by $83.21 \%$ in Amlexanox 5\% Group and $78.57 \%$ in Triamcinolone group ( $p<0.001$ ). Mean number of lesions at baseline was 2.10+1.07 and 2.00+10.12 in Triamcinolone group and Amlexanox respectively. The mean number of lesions was gradually reduced in both groups but there was no significant difference between groups. Mean sizes of the lesions at the baseline, 10 th day, 4 thweek, 8 th week and 12 th week were not significantly different between two groups. The sizes were gradually reduced. Mean percent reduction of sizes of the lesions of two groups were not significantly different at subsequent follow up at 10th day, 4th week, 8th week and 12th week. Conclusion: $5 \%$ Amlexanox is effective only in the field of pain reduction than triamcinolone acetonide, but other effects is nearly similar.
\end{abstract}

Key words: Recurrent Aphthous Ulcer (RAU), 5\% Amlexanox; Triamcinolone acetonide

\section{Introduction}

Recurrent aphthous ulcer (RAU; aphthae; canker sores) is a common condition which is characterized by multiple recurrent small, round or ovoid ulcers with circumscribed margins, erythematous haloes, and yellow or grey floors, appearing first in childhood or adolescence ${ }^{1}$. Recurrent aphthous ulceration (RAU) is the most common disease affecting the oral mucosa. Some $15-20 \%$ of the population is reported to be affected by this condition, with the prevalence as high as $66 \%$ in certain populations ${ }^{2}$. The disease is characterized by painful ulcers affecting the non keratinized mucosa, such as the buccal \& labial mucosa \& the lateral border of the tongue ${ }^{3}$. Recurrent Aphthous Ulcer (RAU) has three different variants- minor aphthous ulcer (MiRAU), major aphthous ulcer (MaRAU) $\&$ herpetiform ulcers 4 . Minor RAU is the common variety affecting about $80 \%$ of RAU patients, characterized by painful round or oval shallow ulcers, regular in outline,

1. Dr. Nasir Uddin Molla, Consultant, Basabo General Hospital, Dhaka

2. Dr. Israt Bhuian, Assistant Professor, Department of Dermatology \& Venereology, Shaheed Suhrawardy Medical College \& Hospital, Dhaka

3. Dr. Shahadat Hossain, Assistant Professor, Department of Dermatology \& Venereology, Shaheed Suhrawardy Medical College \& Hospital, Dhaka

4. Dr. Abdul Wahab, Associate Professor, Department of Dermatology \& Venereology, Shaheed Suhrawardy Medical College \& Hospital, Dhaka

5. Dr. Abu Nayeem, Resident Physician, Shaheed Suhrawardy Medical College Hospital, Dhaka

\section{Correspondence}

Dr. Ishrat Bhuiyan, MBBS, DDV, FCPS, Assistant Professor, Department of Dermatology \& Venereology, Shaheed Suhrawardy Medical College Sher-E-Bangla Nagar, Dhaka; Mobile No: 01711822551; E-mail: ishrat skin@yahoo.com 
less than $10 \mathrm{~mm}$ in diameter with a grey white pseudo membrane surrounded by a thin erythematous halo. It usually occurs on non-keratinized mucosa. The lesions recur at varying frequencies \& heal within 10-14 days without scarring 5 . Major RAU also known as periadenitis mucosa necrotica recurrens occurs in approximately $10 \%$ of RAU patients. The lesions are similar in appearance to those of minor RAU, but they are larger than $10 \mathrm{~mm}$ in diameter, single or multiple \& often heal with scarring. Major RAU usually has its onset after puberty 5 . The third \& least common variety of RAU is herpetic form. This form is characterized by multiple recurrent crops of small painful ulcers that are widely distributed throughout the oral cavity. As many as hundred ulcers may be present at a given time each measuring $2-3 \mathrm{~mm}$ in diameter. They usually heal without scar formation in 7-10 days ${ }^{6}$.

Of these, minor aphthous ulcers are by far the most common, affecting up to $80 \%$ of RAU subjects 5 . Patient may experience a pricking or burning sensation in the mucosa. In this prodromal stage, erythema of the surrounding may be observed or it may appear normal. Within a day or so an oval or round ulcer with a grey white centre \& erythematous halo develops. Typically the ulcers are less than $1 \mathrm{~cm}$ in diameter \& less than five occur at any one time. These ulcers are self limiting \& resolve within 10-14 days without scar formation ${ }^{7}$.

Due to the often uncertain aetiology of RAU \& the unpredictable course of the disease, the primary goals of therapy are to control the pain of the ulcer, promote ulcer healing \& prevent recurrence. Although topical agents do not prevent ulcer recurrence they are arguably the most commonly used treatment modality. A multitude of topical agents are available for symptomatic relief including antibiotics, local anaesthetics, antihistamines, NSAIDS, enzymatic preparations, gamma globulins \& immunosuppressants ${ }^{7}$. However, the problem remains that the efficacy of many of these agents has not been fully evaluated in an adequately designed and controlled clinical trials \& contradictory results are reported in the literature ${ }^{7}$. Of the topical agents that are available for the treatment of RAU-amelxanox, topical corticosteroids $1 \%$ triamcinolone dental paste (Adocortyl or Kenalog in Orabase), 0.05\% Fluocinomide in orabase, betamethasone aerosol. A risk of any of these therapies is oral candidiasis \& a concern is the systemic absorption of the drug through ulcerated mucosa $a^{8,9,10}$. Triamcinolone acetonide is chosen as other therapeutic options are not specifically approved for this condition \& also not readily available in this country. The aim of the present study was to see the efficacy of application of 5\% amelexanox in the treatment of aphthous ulcer in comparison with triamcinolone acetonide.

\section{Methodology}

This study was conducted on 40 patients of age group 18 to 60 years having recurrent aphthous ulcer (RAU) attending at the Department of Dermatology \& Venereology at Shaheed Suhrawardy Medical college Hospital \& Bangabandhu Sheikh Mujib Medical University (BSMMU) \& Department of Dentistry, BSMMU, Dhaka Bangladesh from September 2008 to March 2009. All the cases that were presented with painful oral ulcers with an age of more than 18 years diagnosed clinically and fulfilling the inclusion criteria were enrolled in this study. The patients were randomly allocated into two equal groups which were Group-A \& Group-B. The aims of the study were explained to the patients and informed consent was taken from the patients. Patients' data were recorded in pre-designed structured questionnaire. Data were collected by face to face interview. Information was collected by taking clinical history and clinical examination. Ethical clearance was taken by the local ethical committee. The patients were undergone oral examination, and patients were asked questions regarding any family history of RAS, and socio-demographic parameters. Patients were also taught the way of completion by self-examination, and were given a standardized diary to be competed daily for the entire duration of the study. In the diary, patients were asked to record the presence of ulcers (yes or no) and associated level of pain daily (on a 10 point scale, where 0 indicates no pain and 10, the worst pain ever experiences with mouth ulcers). In addition once during each episode of ulcers, on the day the patient experienced the worst pain, the patient recorded the number of ulcers that were present and their average size. The sizes were assessed by comparing ulcer size to a chart showing 6 circles of increasing diameter between 1 and $10 \mathrm{~mm}$ and numbered 1 to 6 , respectively. Patients were instructed to report after 10 days, 4th week, 8th week and 12th week (end point) to observe the efficacy and side effects of the trial medicine. Subjects with less than two episodes of recurrent aphthous ulcers on the previous year, clinically evident or documented systemic disease, history of a systemic disease that could predispose the patient to recurrent aphthous ulceration, under systemic or topical corticosteroid therapy or immunomodulating treatment and pregnant women or lactating mother were excluded from this study. Amlexanox 5\% oral paste were applied four times daily for 10 days and a follow up were conducted after 10 days, 4th week, 8th week and 12th week to Group-A \& Group-B was given Triamcinolone Acetonide (Kenalog in orabase) oral ointment were applied three times daily for same duration. The diagnosis was made on clinical basis by assessing morphology, site and characters of the lesions. Efficacy was measured by under mentioned scales: Pain 
was measured by the patients marking a $10 \mathrm{~cm}$ line between poles connoting no pain versus severe pain. The visual analogue scale (VAS) and a recorded pain score, number of the lesion, size of the lesion: The size was assessed by comparing ulcer size to a chart showing 6 circles of increasing diameter between 1 and $10 \mathrm{~mm}$ and numbered 1 to 6 respectively. Data were analyzed by computer with the help of SPSS v.17 windows software package.

\section{Result}

Tingling was a complain of $70 \%$ of Triamcinolone group and $80 \%$ of Amlexanox group, $100 \%$ patients of Triamcinolone group and $80 \%$ of Amlexanox group were suffered from pain.

Table 1: Distribution of the patients of both groups by age $(n=40)$

\begin{tabular}{lccr}
\hline $\begin{array}{l}\text { Age } \\
\text { group }\end{array}$ & Group - A & Group- B & p value* \\
\cline { 2 - 3 } & Amlexanox 5\% & $\begin{array}{c}\text { Triamcinolone } \\
\text { acetonide }\end{array}$ & \\
\hline $15-30$ & $10(60.0 \%)$ & $15(75.0 \%)$ & 0.222 \\
$31-45$ & $6(30.0 \%)$ & $4(20.0 \%)$ & \\
$46-60$ & $2(10.0 \%)$ & $1(5.0 \%)$ & \\
Total & $\mathbf{2 0}(\mathbf{1 0 0 . 0} \%)$ & $\mathbf{2 0}(\mathbf{1 0 0 . 0} \%)$ & \\
\hline Mean \pm SD & $\mathbf{3 0 . 6 5} \pm \mathbf{0 . 2 7}$ & $\mathbf{2 7 . 0} \pm \mathbf{8 . 2 3}$ & \\
\hline
\end{tabular}

In Triamcinolone group $40 \%$ had single lesion and $60 \%$ had multiple. There was no significance difference between groups according to these clinical features. At baseline $12(60 \%)$ patients Triamcinolone taker and $8(40 \%)$ of Amlexanox taker had a pain score of $5,40 \%$ of Triamcinolone taker and $60 \%$ of Amlexanox taker had pain score 6 .

Table 2: Distribution of patients of according to sex $(n=40)$

\begin{tabular}{lccc}
\hline \multirow{2}{*}{ Sex } & Group- A & Group- B & p value \\
\cline { 2 - 3 } & Amlexanox 5\% & $\begin{array}{c}\text { Trinmcinolone } \\
\text { acetonide }\end{array}$ & \\
\hline Male & $8(40.0 \%)$ & $12(60.0 \%)$ & 0.206 \\
Female & $12(60.0 \%)$ & $8(40.0 \%)$ & \\
Total & $\mathbf{2 0 ( 1 0 0 . 0 \% )}$ & $\mathbf{2 0}(\mathbf{1 0 0 . 0} \%)$ & \\
\hline
\end{tabular}

There was no significant difference between two groups in terms of baseline pain score. At 10th day follow up Amlexanox group showed better improvement of pain, although there was no significant difference between two groups at 4th week, 8th week and 12th week follow-up. Marked decreased of mean pain score was observed in the patients of both types of treatment. Mean pain score decreased by $83.21 \%$ in Amlexanox 5\% Group and 78.57\% in Triamcinolone group $(\mathrm{p}<0.001)$. Mean number of lesions at baseline was $2.10+1.07$ and $2.00+10.12$ in Triamcinolone group and Amlexanox respectively. The mean number of lesions was gradually reduced in

Table 3: Distribution of the patients by clinical features

\begin{tabular}{lccc}
\hline \multirow{2}{*}{$\begin{array}{c}\text { Clinical } \\
\text { features }\end{array}$} & Group A & Group B & \\
\cline { 2 - 3 } & Amlexanox 5\% & $\begin{array}{c}\text { Triamcinolone } \\
\text { acetonide }\end{array}$ & \\
\hline Tingling & $16(80.0 \%)$ & $14(70.0 \%)$ & 0.465 \\
Pain & $18(90.0 \%)$ & $20(100.0 \%)$ & 0.468 \\
Lesion & & & \\
Solitary & $10(50.0 \%)$ & $8(40.0 \%)$ & 0.525 \\
Multiple & $10(50.0 \%)$ & $12(60.0 \%)$ & \\
\hline
\end{tabular}

both groups but there was no significant difference between groups. Mean sizes of the lesions at the baseline, 10th day, 4th week, 8th week and 12th week were not significantly different between two groups. The sizes were gradually reduced. Mean percent reduction of sizes of the lesions of two groups were not significantly different at subsequent follow up at 10 th day, 4th week, 8th week and 12 th week.

Table 4: Distribution of the patients by intensity of pain based on VAS

\begin{tabular}{lccc}
\hline $\begin{array}{l}\text { Pain (VAS) } \\
\text { rating }\end{array}$ & Amlexanox 5\% & $\begin{array}{c}\text { Triamcinolone } \\
\text { acetonide }\end{array}$ & p value \\
\hline $\begin{array}{l}\text { Baseline } \\
* 5\end{array}$ & $8(40.0)$ & $12(60.0) \#$ & 0.094 \\
$* 6$ & $12(60.0)$ & $8(40.0)$ & \\
At $10^{\text {th }}$ day & & & \\
$* 0$ & $11(55.0)$ & $12(60.0)$ & \\
$* 2$ & $6(30.0)$ & $0(.0)$ & 0.004 \\
$* 3$ & $2(10.0)$ & $0(.0)$ & \\
$* 5$ & $1(5.0)$ & $8(40.0)$ & \\
At $4^{\text {th }}$ weeks & & & \\
$* 0$ & $10(50.0)$ & $4(20.0)$ & \\
$* 5$ & $10(50.0)$ & $16(80.0)$ & 0.047 \\
At $8^{\text {th }}$ weeks & & & \\
$* 0$ & $10(50.0)$ & $4(20.0)$ & 0.047 \\
$* 5$ & $10(50.0)$ & $16(80.0)$ & \\
At $12^{\text {th }}$ weeks & & & \\
$* 0$ & $20(100.0)$ & $18(90.0)$ & \\
$* 5$ & $0(.0)$ & $2(10.0)$ & 0.147 \\
\hline
\end{tabular}

Table 5: Distribution of pain score of different types of aphthae by improvement

\begin{tabular}{lccl}
\hline $\begin{array}{l}\text { Type of } \\
\text { aphthae }\end{array}$ & $\begin{array}{c}\text { Mean pain } \\
\text { score baseline }\end{array}$ & $\begin{array}{c}\text { At 12 } \\
\text { week }\end{array}$ & Improvement \\
\hline Amlexanox 5\% & 5.13 & 0.95 & $83.2 \%$ \\
$\begin{array}{l}\text { Triamcinolone } \\
\text { acetonide }\end{array}$ & 5.71 & 1.71 & $78.6 \%$ \\
\hline
\end{tabular}




\section{Discussion}

The two group has no significant difference in terms of age, sex, socio-economic condition, smoking, site of lesions and baseline clinical features $(\mathrm{p}>0.05)$. Mean age

Table 6: Distribution of the patients by number of lesion on different follow up period

\begin{tabular}{lccl}
\hline Mean \pm SD & Amlexanox 5\% & $\begin{array}{c}\text { Triamcinolone } \\
\text { acetonide }\end{array}$ & P value* \\
\hline Base line & $2.00 \pm 10.12$ & $2.10 \pm 1.07$ & 0.775 \\
10 th day & $0.77 \pm 0.44$ & $0.71 \pm 0.73$ & 0.816 \\
$4^{\text {th }}$ week & $1.33 \pm 0.49$ & $1.22 \pm 0.65$ & 0.618 \\
$8^{\text {th }}$ week & $0.81 \pm 0.06$ & $0.73 \pm 0.68$ & 0.371 \\
$12^{\text {th }}$ week & $0.31 \pm 0.65$ & $0.57 \pm 0.53$ & 0.111 \\
\hline
\end{tabular}

of the patients of triamcinolone group was $30.65 \pm 10.27$ and amlexanox group was $27 \pm 8.23$ years. Out of all respondents in triamcinolone group $60.0 \%$ were male and $40 \%$ were female and $60.0 \%$ female were in amlexanox $5 \%$ group. Chi-square test revealed no statistical significant difference between groups ( $p>0.05)$. None were come from lower socio-economic strata in triamcinolone group as from upper in amlexanox group. Maximum $90.0 \%$ patients from each group were from middle class economic family. No statistical significance difference was observed between groups $(\mathrm{p}>0.05)$. A $25 \%$ of triamcinolone group and $20 \%$ of amlexanox group were smoker with no significant difference between groups. Patients suffering from RAS usually non smokers and there is a lower prevalence and severity of RAS among heavy smokers as opposed to moderate smokers ${ }^{12}$. Some patients report an onset of RAS after smoking cessation, while others report control on re-initiation of smoking. The use of smokeless tobacco also is associated with a significantly lower prevalence of RAS. Nicotine containing tablets also appear to control the frequency of $\mathrm{RAS}^{13}$. There is often a genetic basis for RAS more than $42 \%$ of patients with RAS in Shohat-Zabarski series had first degree relatives with RAS. The likelihood of RAS is $90 \%$ when parents are affected, but only $20 \%$ when neither parent has $\operatorname{RAS}^{14}$ which may support its genetic basis. In this study $22.5 \%$ patients had family history of RAS but association with parental occurrence of disease has not been observed. In triamcinolone group $40 \%$ of lesions are present on oral mucosa, $10 \%$ on tongue and $50 \%$ were on both sides. In amlexanox $5 \%$ group, a $40 \%$ of lesions are present on oral mucosa, $20 \%$ on tongue and $60 \%$ were on both sides. Trauma, stress full life events, foods such as chocolate, coffee, peanuts, cereals, almonds, strawberries, cheese, tomatoes and wheat flour (containing gluten), hormonal imbalance, cessation of smoking sometimes act as predisposing factors ${ }^{15}$.
In this study $21(70.0 \%)$ cases had history of trauma before development of aphthous ulcer, $18(60 \%)$ cases had stress full life event, $13(43.3 \%)$ cases had given history of involvement of some food, $3(10 \%)$ cases had given history of their cessation of smoking. Of all female patients $2(33.3 \%)$ cases had given history of relationship of their aphthous ulcer and menstrual cycle. Out of all 20 patients of triamcinolone group tingling sensation was found in $70 \%$ patients and pain in $90.0 \%$ patients. A $40 \%$ of triamcinolone acetonide users and $50 \%$ of amlexanox used had solitary lesion. Baseline mean number of lesions was $2.1 \pm 1.07$ and $2.00 \pm 10.12$ and at 12 th week it was $0.57 \pm 0.53$ and $0.31 \pm 0.65$ in triamcinolone and amlexanox group respectively. The improvement was significant difference between two groups.

Table 7: Distribution of the respondents by size of the lesions

\begin{tabular}{|c|c|c|c|}
\hline $\begin{array}{l}\text { Size of the } \\
\text { lesions }\end{array}$ & $\begin{array}{l}\text { Amelxanox } \\
\text { Mean + SD }\end{array}$ & $\begin{array}{c}\text { Triamcinolone } \\
\text { Mean + SD }\end{array}$ & $P$ value \\
\hline At Base line & $3.66 \pm 0.72$ & $3.71 \pm 0.27$ & 0.19 \\
\hline At $10^{\text {th }}$ day & $0.17 \pm 0.07$ & $0.23 \pm 0.105$ & 0.06 \\
\hline At $4^{\text {th }}$ week & $0.74 \pm 0.02$ & $1.96 \pm 0.74$ & 0.43 \\
\hline At $8^{\text {th }}$ week & $0.41 \pm 0.131$ & $0.53 \pm 0.33$ & 0.52 \\
\hline At $12^{\text {th }}$ week & $0.17 \pm 0.07$ & $0.23 \pm 0.105$ & 0.06 \\
\hline $\begin{array}{l}\text { Reduction size at } \\
10^{\text {th }} \text { day } \\
\text { Follow up from } \\
\text { baseline }(\%)\end{array}$ & $93.80 \pm 21.07$ & $95.05 \pm 13$ & 0.09 \\
\hline $\begin{array}{l}\text { Reduction of size } \\
\text { at } 8^{\text {th }} \text { week from } \\
\text { baseline } \%\end{array}$ & $79.78 \pm 7.56$ & $68.12 \pm 11.93$ & 0.81 \\
\hline $\begin{array}{l}\text { Reduction of size } \\
\text { at } 8^{\text {th }} \text { week from } \\
\text { baseline }(\%)\end{array}$ & $91.49 \pm 19.04$ & $88.07 \pm 17.65$ & 0.53 \\
\hline $\begin{array}{l}\text { Reduction of size } \\
\text { at } 12^{\text {th }} \text { week } \\
\text { from baseline }(\%)\end{array}$ & $93.80 \pm 21.07$ & $95.07 \pm 13$ & 0.09 \\
\hline
\end{tabular}

*Significant $(\mathrm{p}<0.05)$

Mean baseline pain score in the triamcinolone group was 5.13 and it reduced to 1.71 at $12^{\text {th }}$ week. Marked decreased of mean pain score was observed in the patients of both types of treatment. Mean pain score decreased by $83.2 \%$ in amlexanox $5 \%$ group and $78.6 \%$ in triamcinolone group $(p<0.001)$. In the light of these findings it is evident that $5 \%$ amlexanox is effective in the treatment of recurrent aphthous stomatitis and is effective as topical triamcinolone, except slightly faster pain relieve by amlexanox. Geer et $\mathrm{al}^{9}$ noted the efficacy of amlexanox in the treatment of RAS in a double blind study, they did not use any control. The study by Robert et $\mathrm{al}^{16}$ and Khandewell et al ${ }^{11}$ use placebo or vehicle control and 
proved the efficacy of $5 \%$ amlexanox in RAS. Geer et al ${ }^{9}$ after a double-blind study of amlexanox on 32 patients of aphthous ulcer, concluded that amlexanox on 32 patients of aphthous ulcer, concluded that amlexanox is effective in reducing aphthous ulcer erythema, pain and lesion size ${ }^{8}$ and reported that global improvement of aphthous ulcer was seen in $98 \%$ of those patients treated with $5 \%$ amlexanox paste ${ }^{15}$. In this study ulcer size was reduced by $93.80 \pm 21.07$ which is not significantly different from triamcinolone group.

In current study, some improvement of Visual Analogue Scale (VAS) reducing (pain reduction) was observed among amlexanox group than triamcinolone acetonide group but its other effects is nearly similar to topical triamcinolone acetonide. There is no such type of study in this country comparing the efficacy of topical amelexanox with topical triamcinolone, against which this finding can be weighted.

\section{Conclusion}

In current study no adverse events were experienced by any of the modalities. So we conclude that $5 \%$ amlexanox is effective only in the field of pain reduction than triamcinolone acetonide for recurrent aphthous stomatitis (RAS).

\section{References}

1. Jurge S, Kuffer R, Scully C, Porter SR. 'Recurrent aphthous stomatitis' Oral Diseases. 2006;12:1-21

2. Axell T, Henricsson V. "Association between recurreny aphthous ulcers \& habits", Scand J Dent Res, 1985;93:29-43

3. Bagan J V, Sarchis JM, Milian M A, Penarrocha M, Silves trr FJ. "Recurrent aphthous stomatitis : A study of the clinical characteristics of lesions in 93 cases' J ral pathol Med.1991;20:395-7

4. Andrews Diseases of the skin, clinical darmatology, william D. James 11 th edition. Page- 797-798.

5. Porter SR, Scully C, Flint S Orofacial Manifestations in Primary immunodeficiencies involving IgA deficiency. J Oral Pathol Med 1993;22: 117-119

6. Natan SS, hayrinen- Immonen R, Hietanen Jet al, Increased density of lymphocytes bearing gamma /delta T-cell receptors in recurrent aphthous ulceration (RAU), Int J Oral Maxillo fac surg 2000;29:379-380

7. Wool SB \& Somis ST, Recurrent aphthous stomatitis: clinical characteristics \& associated systemic disorders; Semin Cutan Med Surg. 1996;16:278-283

8. Rogers RS. 'Recurrent aphthous stomatitis: clinical characteristics and associated systemic disorders', Semin Cutan Med Surg.1997;16:278-83

9. Greer RO Jr. Lindenmuth JE, Juarez T, Khandwala A. 'A double- blind study of topically applied $5 \%$ amlexanox in the treatment of aphthous ulcers' ' J Oral Maxillofac Surg. 1993;51 (3243) :248-9

10. Binnie WH, Curro FAKhandwala A, Van Inwegan RG Amelaxanox oralpaste: a novel tretment that accerlerates the healing of aphthous ulcers. Compend Contin Educ Dent 1997;48: 1116-1124

11. Khandwala A, Van inwegen RG, Alfano MC. '5\% amlexanox oral paste, aew treatment for recurrent minor aphthous ulcers: I. clinical demonstration of acceleration of healing and resolution of pain, Oral Surg Oral Med Oral Pathol Oral Radiol Endod. 1997; 83, (2):222-30.
12. SB \& Sonis ST Recurrent Aphthous Ulcers: a review of diagnosis \& treatment " Jam Dent Assoct 1996; 127:1202-1213

13. Tuzun B, Wolf R, Tuzun $Y$, Serdaroglu $S$, Recurrent aphthous stomatitis and smoking. Int J Dermatol 2000; 39: 358-360.

14. Scully C, Gorsky M and Lozada-Nur F, T, 'The diagnosis and management of recurrent aphthous stomatitis A consensus approach', JADA. 2008 Vol. 134.

15. Ship II. 'Epidemiologic aspects of recurrent aphthous ulcerations', Oral Surg Oral Med Oral Pathol. 1972;33:400-406.

16. Robert OG, James EL, Juarez T. 'A double-blind study of topically applied 5\% amlexanox in the treatment of aphthous ulcers', J Oral Maxillofac Surg. 1993;51:243-248. 